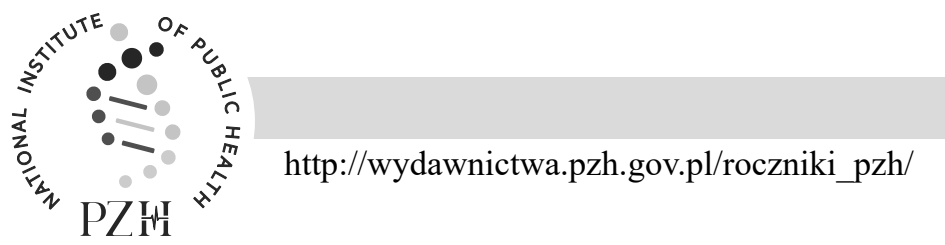

\title{
URIC ACID ALTERATIONS BY CONSUMPTION OF GLUTEN-FREE BAKERY PRODUCTS IN RELATION TO CARDIOVASCULAR AND METABOLIC SYNDROME RISK FACTORS
}

\author{
Martina Gažarovál, Lucia Mečiarovál
}

${ }^{1}$ Slovak University of Agriculture, Faculty of Agrobiology and Food Resources, Department of Human Nutrition, Tr. A. Hlinku 2, 94976 Nitra, Slovak Republic

\begin{abstract}
Background. Serum uric acid (UA) levels are one of the determinants of the cardiovascular disease and metabolic syndrome but none of criteria for that syndrome include serum UA. Consumption of bakery products (with or without gluten) is associated with an increasing prevalence of overweight/obesity and hyperuricemia frequently occurs in subjects with overweight and obesity.

Objective. The aim of the study was to find out how 6-weeks consumption of gluten-free bakery products can affect risk factors for cardiovascular disease and metabolic syndrome, and especially uric acid levels.

Material and Methods. The group was composed of 27 female volunteers consuming gluten-free bakery products during 6-week period. The biochemical parameters levels were measured by Biolis 24i Premium, the anthropometric parameters by InBody 720 and blood pressure by OMRON Microlife.

Results. We found a non-significant increase in total cholesterol and decrease in triglycerides, in the case of LDL cholesterol a significant reduction in values and increase of HDL cholesterol. Glucose level increased significantly, but uric acid has not changed significantly. We found the highest total cholesterol, triglyceride, and LDL concentrations in the third UA quartile. The highest glucose concentrations were found in the lower UA quartiles, while the lowest in the highest quartiles. Linear increases in UA concentrations were not observed in any of the parameters. Evaluation of the anthropometric parameters showed that while values of BMI, VFA, fat mass and waist circumference were the highest at the beginning of the study in the second quartile, after intervention the highest values were shifted to the third quartile. Conclusions. Due to the consumption of gluten-free bakery products the risk values of the monitored parameters shifted to higher UA quartiles.
\end{abstract}

Key words: uric acid, gluten-free, lipid profile, blood pressure, weight gain rate

\section{INTRODUCTION}

Uric acid (UA) is the end-product of purine metabolism in humans [53]; product of an exogenous pool of purines and endogenous purine metabolism [14]. Its levels vary significantly within humans as the results of the factors that increase generation (e.g. high purine or protein diet) or decrease excretion (e.g. reduction in glomerular filtration rate) [55]. The production and catabolism of purines are relatively constant between 300 and $400 \mathrm{mg}$ per day [14]. Exogenous sources that can increase serum UA include fatty meat, organ meat, and seafood [33] and fructose is another source of exogenous UA. Higher serum UA levels are found in men (with older age, higher blood pressure, increasing cholesterol level and creatinine, and higher body mass index) $[24,36]$.

Hyperuricemia is usually defined as 6.5 or 7.0 mg.dL $\mathrm{dL}^{-1}$ in men and $6.0 \mathrm{mg} . \mathrm{dL}^{-1}$ in women. This condition frequently occurs in subjects with overweight and obesity, insulin resistance, glucose intolerance, dyslipidemia, hypertension, fatty liver, etc. [23, 60].

Some observational studies have suggested that serum UA levels are one of the determinants of the metabolic syndrome and cardiovascular diseases [4]. Metabolic syndrome has become health problem worldwide due to its relationships with cardiovascular disease and type 2 diabetes. It rises with aging and women are more affected than men [50]. The diagnostic criteria for metabolic syndrome vary $[3,28]$, but three or more of these manifestations are needed to diagnose:

Corresponding author: Martina Gažarová, Ing. PhD (https://orcid.org/0000-0001-8275-7311) Slovak University of Agriculture, Faculty of Agrobiology and Food Resources, Department of Human Nutrition, Tr. A. Hlinku 2, 94901 Nitra, Slovakia, phone: +42137 641 4210, e-mail: martina.gazarova@uniag.sk; martina.gazarova@gmail.com 
for European individuals waist circumference $\geq 94$ and $\geq 80 \mathrm{~cm}$ in men and women respectively; serum triglycerides $1.7 \mathrm{mmol} . \mathrm{L}^{-1}$; high-density lipoprotein cholesterol $<1 \mathrm{mmol} . \mathrm{L}^{-1}$ and $1.3 \mathrm{mmol} . \mathrm{L}^{-1}$ in men and women respectively; blood pressure $\geq 140 / 90 \mathrm{mmHg}$; and fasting blood glucose $>6.1 \mathrm{mmol}^{-1} \mathrm{~L}^{-1}[2,3,8]$. None of these criteria include serum UA although elevated levels are in subjects with metabolic syndrome [62] and it has therefore been suggested that serum UA is one of the determinants of syndrome [46]. Whilst hyperuricemia is not accepted as factor for the diagnosis of metabolic syndrome, many studies have suggested that serum UA levels are uniformly elevated $[11,47]$. Body composition including muscle mass and fat mass are associated with serum UA levels. In adults with normal body mass index, the metabolic syndrome is 10 times higher in those having serum UA $\geq 10$ mg.dL ${ }^{-1}$ compared to those with $<6 \mathrm{mg}^{-\mathrm{dL}^{-1}}$ [17]. The hazard ratio of incident metabolic syndrome shows a steady increase when normal adults were allocated into four quartiles according to serum UA [63]. Shani et al. [52] found that high normal serum UA was also associated with future development of type 2 diabetes among lean healthy and normoglycemic women. Elevated serum UA predicted diabetes mellitus and insulin resistance [35]. Uric acid is also commonly associated with hypertension and the risk to develop the hypertension rises greater in hyperuricemic male and female subjects; this chance augments in older age [58, 61]. Leiba et al. [39] found that those with serum UA higher than $3 \mathrm{mg} \cdot \mathrm{dL}^{-1}$ had a greater chance to develop hypertension and the higher the serum UA within the normal range, the greater was the risk to develop hypertension. The risk is stronger in younger ages and in females and high serum UA is one of the major predictors of worse blood pressure control [18, $38,56]$. Increased serum UA was also appointed as independent risk factor for overall and cardiovascular mortality. The relationship is higher in the lowest and highest quintiles in both men and women [59].

Gluten-free eating patterns are frequently perceived to be healthier than gluten-containing ones and good health is the primary reason given for a gluten-free diet adoption in healthy population. In recent years there has been an increasing interest on gluten-free foodstuffs [34]. The proportion of people on a gluten-free diet exceeds in more than five times the number of those who require gluten exclusion as a treatment [13]. However, the nutritional value and quality of gluten-free products is questionable. Lucisano et al. [41] reported that most gluten-free products had poor cooking quality compared with their wheat counterparts and there is a need to improve the nutritional quality of these products. Researchers found an excess intake of total energy, animal protein and fat and a lower intake of dietary fibre, magnesium and folic acid by following a glutenfree diet $[44,66]$. Gluten-free breads had a higher glycaemic index than the conventional breads and had great divergences in fat [19]. Almost all the glutenfree products were high in available carbohydrates [42]. Gluten-free bread provides twice as much fat, mainly saturated fat in comparison to its equivalents with gluten [44]. Consumption of bread and bakery products (with or without gluten) is associated with an increasing prevalence of overweight and obesity. It was demonstrated that the obesity risk is increased in celiac on gluten-free diet because of the high glycemic index of the gluten-free diet [37].

Therefore, the aim of the study was to find out how six weeks of consumption of gluten-free bakery products can affect selected risk factors for cardiovascular disease and metabolic syndrome, and especially uric acid, whose elevated levels are a significant predictor of these diseases.

\section{MATERIALS AND METHODS}

\section{Characteristics of the participants}

Twenty-seven female volunteers were included in the study. The requirement for participation was the consent of individuals with whole study and measurement conditions which they will have to complete during the research. The group of participants was composed of volunteers from the general population, consuming gluten-free bread and glutenfree bakery products during 6-week period; however the participants of the study were not allowed to be on total and strict gluten-free diet. Participants with the present severe disease or with recommended special dietary regimen were excluded prior to the start of the study. The amount of bread and bakery products was determined according to the recommended dietary allowance for the Slovak population 150-200 grams per day. All participants were asked not to change their eating habits and also not to change their habits related to the physical activity. Volunteers completed a total of 2 measurements $\left(1^{\text {st }}\right.$ measurement before consumption as a control, $2^{\text {nd }}$ measurement after the 6 -week consumption). The trial was approved by the Ethic Committee at the Specialized St. Svorad Hospital Nitra Zobor (Slovakia); (protocol no. 012911/2016).

\section{Dietary Assessment}

For study purposes, we monitored the nutritional intake (Table 1) of study participants in order to evaluate the recommended nutritional doses and to better assess the potential impact of consumption of gluten-free bakery products on the blood concentrations of the monitored parameters. Dietary intake was assessed using 3-day 24-h food recalls, two on weekdays and one at the weekend. We used the 
Table 1. Nutrient intake of participants

\begin{tabular}{|c|c|c|c|c|}
\hline & Energy $(\mathrm{kJ})$ & Carbohydrates (g) & Lipids (g) & Proteins $(\mathrm{g})$ \\
\hline mean $\pm \mathrm{SD}$ & $7840 \pm 3241$ & $229.54 \pm 113$ & $79.34 \pm 37$ & $68.75 \pm 28$ \\
\hline $\max$ & 17290.35 & 643.82 & 208.42 & 161.33 \\
\hline $\min$ & 2378.35 & 66.75 & 25.11 & 21.2 \\
\hline med & 7218.25 & 195.54 & 69.7 & 66.09 \\
\hline \multirow[t]{2}{*}{$\bmod$} & ND & ND & 93.48 & ND \\
\hline & $\begin{array}{c}\text { Dietary fibre } \\
(\mathrm{g})\end{array}$ & $\begin{array}{l}\text { Polyunsaturated } \\
\text { fatty acids (g) }\end{array}$ & $\begin{array}{l}\text { Monounsaturated } \\
\text { fatty acids (g) }\end{array}$ & $\begin{array}{c}\text { Saturated } \\
\text { fatty acids (g) }\end{array}$ \\
\hline mean $\pm \mathrm{SD}$ & $19.04 \pm 11$ & $11.57 \pm 6$ & $22.06 \pm 11$ & $24.92 \pm 14$ \\
\hline $\max$ & 58.45 & 36.38 & 53.34 & 63.66 \\
\hline $\min$ & 4.8 & 1.13 & 5.84 & 7.8 \\
\hline med & 17.72 & 10.3 & 19.1 & 21.39 \\
\hline \multirow[t]{2}{*}{$\bmod$} & 58.45 & 13.3 & 36.07 & 47.48 \\
\hline & Phosphorus (mg) & Magnesium (mg) & Sodium (mg) & Iron (mg) \\
\hline mean \pm SD & $1026.35 \pm 501$ & $335.89 \pm 541$ & $3974.77 \pm 2772$ & $12.65 \pm 7$ \\
\hline $\max$ & 2923.66 & 5004.57 & 15147.43 & 36.44 \\
\hline $\min$ & 162.22 & 78.94 & 967.53 & 3.33 \\
\hline med & 909.75 & 258.12 & 3248.25 & 11.36 \\
\hline \multirow[t]{2}{*}{$\bmod$} & ND & ND & ND & 36.44 \\
\hline & Calcium (mg) & Potassium (mg) & Pyridoxine (mg) & Thiamine (mg) \\
\hline mean $\pm \mathrm{SD}$ & $775.62 \pm 414$ & $2332.23 \pm 1148$ & $1.20 \pm 1$ & $1.02 \pm 1$ \\
\hline $\max$ & 2111.8 & 6024.09 & 4.23 & 3.8 \\
\hline $\min$ & 185.87 & 675.95 & 0.34 & 0.14 \\
\hline med & 747.32 & 2055.11 & 1.12 & 0.94 \\
\hline $\bmod$ & ND & ND & 1.32 & 0.93 \\
\hline
\end{tabular}

Table 2. Compliance with the standard for nutrient intake (according to Kajaba et al. [32])

\begin{tabular}{|c|c|c|c|c|}
\hline & $\begin{array}{c}\text { Energy } \\
(\%)\end{array}$ & $\begin{array}{c}\text { Carbohydrates } \\
(\%)\end{array}$ & $\begin{array}{l}\text { Lipids } \\
(\%)\end{array}$ & $\begin{array}{l}\text { Proteins } \\
(\%)\end{array}$ \\
\hline mean & 89 & 63 & 124 & 133 \\
\hline $\max$ & 503 & 183 & 321 & 316 \\
\hline \multirow[t]{2}{*}{$\min$} & 25 & 18 & 39 & 41 \\
\hline & Dietary fibre (\%) & $\begin{array}{l}\text { Polyunsaturated } \\
\text { fatty acids }(\%)\end{array}$ & $\begin{array}{l}\text { Monounsaturated } \\
\text { fatty acids }(\%)\end{array}$ & $\begin{array}{c}\text { Saturated } \\
\text { fatty acids }(\%)\end{array}$ \\
\hline mean & 81 & 55 & 104 & 116 \\
\hline $\max$ & 244 & 182 & 267 & 318 \\
\hline \multirow[t]{2}{*}{$\min$} & 5 & 7 & 26 & 36 \\
\hline & Phosphorus (\%) & $\begin{array}{l}\text { Magnesium } \\
(\%)\end{array}$ & $\begin{array}{l}\text { Sodium } \\
\qquad \%)\end{array}$ & $\begin{array}{r}\text { Iron } \\
(\%)\end{array}$ \\
\hline mean & 87 & 83 & 256 & 78 \\
\hline $\max$ & 244 & 203 & 939 & 243 \\
\hline \multirow[t]{2}{*}{$\min$} & 21 & 23 & 65 & 21 \\
\hline & $\begin{array}{l}\text { Calcium } \\
(\%)\end{array}$ & $\begin{array}{c}\text { Potassium } \\
(\%) \\
\end{array}$ & $\begin{array}{c}\text { Pyridoxine } \\
(\%)\end{array}$ & $\begin{array}{c}\text { Thiamine } \\
(\%)\end{array}$ \\
\hline mean & 85 & 52 & 67 & 100 \\
\hline $\max$ & 746 & 134 & 240 & 280 \\
\hline $\min$ & 19 & 15 & 19 & 14 \\
\hline
\end{tabular}


nutritional software program Mountberry - Nutrition \& Fitness Software (2011, Version 1.1, Slovakia). Mountberry provides a complete analysis of food, meals, recipes based on an updated food database and nutritional recommendations for nutrient intake, health insights, dietary guidelines, and individual user needs. The average nutrient intake for participants was assessed according to the Recommended Dietary Allowance (OVD in Slovakia) updated in 2015 [32] (Table 2). During the trial we focused on basic parameters such as energy, proteins, carbohydrates, fats and also minerals (phosphorus, magnesium, sodium, iron, calcium and potassium) and vitamins (pyridoxine and thiamine).

\section{Blood samples}

Blood samples were obtained before and after intervention. Venous blood was collected in the morning after $8 \mathrm{~h}$ of fasting using $2.5 \mathrm{~mL}$ EDTA solution and in a $2 \times 7.5 \mathrm{~mL}$ serum gel tube. After the separation of blood serum, the parameters levels were measured by automatic biochemical analyser Biolis 24i Premium (Tokyo Boeki Machinery Ltd., Japan) using direct ion selective electrodes methods in the laboratory of the Department of Human Nutrition (Slovak University of Agriculture in Nitra). We focused on changes in serum lipid profile (total cholesterol, triglycerides, LDL cholesterol and HDL cholesterol), glucose and uric acid. The serum UA concentration was divided into four quartiles as follows: $1^{\text {st }}$ quartile $\leq 3.5 \mathrm{mg} . \mathrm{dL}^{-1}, 2^{\text {nd }}$ quartile $3.6-4 \mathrm{mg} \cdot \mathrm{dL}^{-1}, 3^{\text {rd }}$ quartile 4.1-4.6 mg.dL ${ }^{-1}$ and $4^{\text {th }}$ quartile $\geq 4.7 \mathrm{mg} . \mathrm{dL}^{-1}[63]$.

\section{Anthropometric measurements}

Body height was measured in a standing position without shoes on the electronical medical scales Tanita WB-300 while shoulders were in normal alignment and the data were recorded to the nearest $0.1 \mathrm{~cm}$. Weight was measured in light clothing without shoes using a standard scale and recorded to the nearest 0.1 $\mathrm{kg}$. Waist circumference was measured at the umbilical level and that of the hip at the maximum level over light clothing, using a stretched tape meter, without any pressure to body surface and measurements were recorded to the nearest $0.1 \mathrm{~cm}$. BMI $\left(\mathrm{kg} \cdot \mathrm{m}^{-2}\right)$ was calculated as weight $(\mathrm{kg})$ divided by square of the height $\left(\mathrm{m}^{2}\right)$.

The anthropometric measurements were made by using InBody 720 (Biospace Co. Ltd., Seoul, Republic of Korea). Body composition was diagnosed by multifrequency bioelectrical impedance analysis, which measures the total impedance at frequencies of 1, 5, 50, 100, 500, $1000 \mathrm{kHz}$. Each of the participants was informed with the measurement procedure, explained the possible risks of measuring in the case of pregnancy or having an artificial pacemaker at the heart. Before the measurement, participants were asked to excrete and refrain from drinking excessive amounts of water. At the same time each participant signed informed consent for the measurement procedure and also agreed to the processing of personal data. The Lookin'Body 3.0 software was used to process the results. We focused especially on visceral fat area $\left(\mathrm{VFA}, \mathrm{cm}^{2}\right.$ ) and fat mass (FM, $\mathrm{kg} / \%$ ).

\section{Measurement of blood pressure}

Blood pressure (systolic and diastolic) was measured by using OMRON Microlife AG, 9443 (Widnau/Switzerland) with fully automatic operation and the possibility of using both the classic and elongated inflatable cuff on the arm. Blood pressure was measured after the body fluid had settled, resting, sitting. The reference limits were for systolic pressure $120-129 \mathrm{mmHg}$, diastolic pressure $80-84 \mathrm{mmHg}$ and pulse $60-90$ beats per minute.

\section{Statistical analysis}

We evaluated the collected data from the measurements statistically and graphically in Microsoft Office Excel 2010 (Los Angeles, CA, USA). The changes between biochemical and anthropometric measurements were performed using paired Student's $t$-test and the data were presented as mean \pm standard deviation (SD). Statistical analyses were performed using the program STATISTICA Cz version 10. Differences among data were also tested with a one-way analysis of variance (ANOVA) and were compared using Tukey's Post Hoc Test. The levels of statistical significance were set at $P<0.05\left(^{*}\right)$, $P<0.01$ (**), $P<0.001$ (***). We used also Pearson's correlation analysis between parameters.

\section{RESULTS AND DISCUSSION}

Changes of the biochemical and anthropometric parameters after intervention are shown in Table 3. Within the lipid spectrum, we found a non-significant increase in total cholesterol from $5.82 \pm 0.94$ mmol. $\mathrm{L}^{-1}$ to $5.93 \pm 0.91 \mathrm{mmol} . \mathrm{L}^{-1}$ and a decrease in triglycerides from $0.98 \pm 0.41 \mathrm{mmol} . \mathrm{L}^{-1}$ to $0.91 \pm 0.46 \mathrm{mmol} . \mathrm{L}^{-1}$. However, in the case of LDL cholesterol, we found a significant reduction in values from $3.29 \pm 0.75$ mmol.L $\mathrm{L}^{-1}$ to $2.97 \pm 0.71 \mathrm{mmol.L} \mathrm{L}^{-1}(P<0.001)$ and increase of HDL cholesterol from $1.93 \pm 0.40 \mathrm{mmol}^{-L^{-1}}$ to $2.02 \pm 0.41 \mathrm{mmol}^{-\mathrm{L}^{-1}}(P<0.05)$. In this regard, we can conclude that an increase in total cholesterol may have been due to an increase in HDL, but it is important for the assessment of cardiovascular risk in which HDL and LDL subfractions are predominant, since not every increase in HDL or decrease in LDL must clearly predict increased or decreased cardiovascular risk. Therefore, further analyses are needed. 
Table 3. Changes of the biochemical and anthropometric parameters after intervention

\begin{tabular}{|c|c|c|c|c|c|c|c|}
\hline \multirow{2}{*}{ Parameters } & \multicolumn{3}{|c|}{ Baseline } & \multicolumn{3}{|c|}{ After 6 weeks } & \multirow[b]{2}{*}{$P$ value } \\
\hline & mean $\pm \mathrm{SD}$ & $\max$ & $\min$ & mean $\pm \mathrm{SD}$ & $\max$ & $\min$ & \\
\hline Age (years) & $29.11 \pm 7$ & 45 & 23 & & & & \\
\hline Height (cm) & $167.1 \pm 5.6$ & 176 & 156 & & & & \\
\hline Weight (kg) & $63.51 \pm 11.93$ & 101.4 & 48.5 & $63.29 \pm 11.88$ & 100.5 & 48.1 & 0.1550 \\
\hline Uric acid $\left(\mathrm{mg} \cdot \mathrm{dL}^{-1}\right)$ & $3.7 \pm 0.72$ & 5.28 & 2.55 & $3.71 \pm 0.81$ & 5.48 & 2.38 & 0.9293 \\
\hline Total cholesterol $\left(\mathrm{mmol} . \mathrm{L}^{-1}\right)$ & $5.82 \pm 0.94$ & 8.50 & 4.41 & $5.93 \pm 0.91$ & 7.94 & 4.71 & 0.3066 \\
\hline Triglycerides $\left(\mathrm{mmol} . \mathrm{L}^{-1}\right)$ & $0.98 \pm 0.41$ & 2.05 & 0.48 & $0.91 \pm 0.46$ & 2.63 & 0.48 & 0.2595 \\
\hline LDL cholesterol $\left(\mathrm{mmol} . \mathrm{L}^{-1}\right)$ & $3.29 \pm 0.75$ & 5.10 & 1.78 & $2.97 \pm 0.71$ & 4.55 & 1.75 & $<0.001$ \\
\hline HDL cholesterol $\left(\mathrm{mmol} . \mathrm{L}^{-1}\right)$ & $1.93 \pm 0.40$ & 2.95 & 1.18 & $2.02 \pm 0.41$ & 2.97 & 1.27 & 0.0166 \\
\hline Glucose $\left(\mathrm{mmol} . \mathrm{L}^{-1}\right)$ & $4.55 \pm 0.39$ & 5.23 & 3.89 & $4.85 \pm 0.51$ & 5.84 & 3.92 & $<0.001$ \\
\hline Body mass index $\left(\mathrm{kg} \cdot \mathrm{m}^{-2}\right)$ & $22.75 \pm 4.12$ & 35.50 & 17.75 & $22.67 \pm 4.13$ & 35.19 & 17.68 & 0.1797 \\
\hline Visceral fat area $\left(\mathrm{cm}^{2}\right)$ & $74.10 \pm 30.16$ & 153.94 & 37.80 & $74.77 \pm 31.37$ & 167.22 & 41.07 & 0.3732 \\
\hline Fat mass $(\mathrm{kg})$ & $18.70 \pm 9.44$ & 51.30 & 8.70 & $18.66 \pm 9.27$ & 50.50 & 9.80 & 0.8080 \\
\hline Percentage of body fat (\%) & $28.16 \pm 8.02$ & 50.59 & 16.51 & $28.22 \pm 7.70$ & 50.21 & 19.10 & 0.8155 \\
\hline Waist circumference $(\mathrm{cm})$ & $82.08 \pm 10.99$ & 110.30 & 68.40 & $82.31 \pm 11.30$ & 114.90 & 69.90 & 0.4023 \\
\hline Systolic blood pressure $(\mathrm{mmHg})$ & $121 \pm 9.82$ & 141 & 103 & $119 \pm 9.82$ & 142 & 101 & 0.2138 \\
\hline Diastolic blood pressure $(\mathrm{mmHg})$ & $79 \pm 9.74$ & 97 & 64 & $77 \pm 8.61$ & 94 & 63 & 0.3363 \\
\hline
\end{tabular}

Table 4. Changes of the biochemical and anthropometric parameters according to quartiles of serum uric acid concentration

\begin{tabular}{|c|c|c|c|c|c|c|}
\hline \multirow{2}{*}{ UA quartiles } & \multicolumn{2}{|c|}{ UA (mg.dL $\left.{ }^{-1}\right)$} & \multicolumn{2}{|c|}{ T-C (mmol.L-1) } & \multicolumn{2}{|c|}{ TAG (mmol.L-1) } \\
\hline & baseline & after 6 weeks & baseline & after 6 weeks & baseline & after 6 weeks \\
\hline$<3.5{\mathrm{mg} . \mathrm{dL}^{-1}}^{-1}$ & 3.0 & 3.0 & 5.64 & 5.72 & 0.92 & 0.81 \\
\hline $3.6-4 \mathrm{mg} \mathrm{dL}^{-1}$ & 3.7 & 3.9 & 5.62 & 5.68 & 1.02 & 0.86 \\
\hline $4.1-4.6 \mathrm{mg} . \mathrm{dL}^{-1}$ & 4.3 & 4.4 & 6.39 & 6.72 & 1.09 & 1.26 \\
\hline$>4.7 \mathrm{mg} \mathrm{dL}^{-1}$ & 5.0 & 5.0 & 5.76 & 6.20 & 0.93 & 0.94 \\
\hline \multirow{2}{*}{ UA quartiles } & \multicolumn{2}{|c|}{ LDL (mmol.L $\left.\mathrm{L}^{-1}\right)$} & \multicolumn{2}{|c|}{ HDL (mmol.L-1) } & \multicolumn{2}{|c|}{$\mathrm{G}\left(\mathrm{mmol} . \mathrm{L}^{-1}\right)$} \\
\hline & baseline & after 6 weeks & baseline & after 6 weeks & baseline & after 6 weeks \\
\hline$<3.5 \mathrm{mg} . \mathrm{dL}^{-1}$ & 3.24 & 2.80 & 1.83 & 2.00 & 4.53 & 5.01 \\
\hline $3.6-4 \mathrm{mg} \mathrm{dL}^{-1}$ & 3.39 & 2.60 & 1.73 & 2.08 & 4.66 & 4.53 \\
\hline 4.1-4.6 mg.dL $\mathrm{dL}^{-1}$ & 3.48 & 3.77 & 2.23 & 1.97 & 4.52 & 5.13 \\
\hline$>4.7 \mathrm{mg} . \mathrm{dL}^{-1}$ & 2.88 & 3.29 & 2.11 & 2.05 & 4.43 & 4.55 \\
\hline \multirow{2}{*}{ UA quartiles } & \multicolumn{2}{|c|}{ BMI $\left(\mathrm{kg} \cdot \mathrm{m}^{-2}\right)$} & \multicolumn{2}{|c|}{ VFA $\left(\mathrm{cm}^{2}\right)$} & \multicolumn{2}{|c|}{ FM (kg) } \\
\hline & baseline & after 6 weeks & baseline & after 6 weeks & baseline & after 6 weeks \\
\hline$<3.5{\mathrm{mg} . \mathrm{dL}^{-1}}^{-1}$ & 22.37 & 22.36 & 70.91 & 71.49 & 17.25 & 17.66 \\
\hline $3.6-4 \mathrm{mg} \cdot \mathrm{dL}^{-1}$ & 24.21 & 21.13 & 86.82 & 65.52 & 23.16 & 15.50 \\
\hline $4.1-4.6 \mathrm{mg} . \mathrm{dL}^{-1}$ & 22.46 & 26.34 & 66.98 & 101.30 & 17.10 & 27.70 \\
\hline$>4.7{\mathrm{mg} . \mathrm{dL}^{-1}}^{-1}$ & 21.33 & 22.35 & 70.34 & 72.80 & 16.80 & 17.60 \\
\hline \multirow{2}{*}{ UA quartiles } & \multicolumn{2}{|c|}{$\mathrm{WC}(\mathrm{cm})$} & \multicolumn{2}{|c|}{ SBP (mmHg) } & \multicolumn{2}{|c|}{ DBP (mmHg) } \\
\hline & baseline & after 6 weeks & baseline & after 6 weeks & baseline & after 6 weeks \\
\hline$<3.5 \mathrm{mg} . \mathrm{dL}^{-1}$ & 81.4 & 81.2 & 119 & 118 & 77 & 75 \\
\hline 3.6-4 mg.dL ${ }^{-1}$ & 83.30 & 78.40 & 120 & 117 & 73 & 78 \\
\hline 4.1-4.6 mg.dL ${ }^{-1}$ & 79.25 & 92.9 & 124 & 126 & 83 & 80 \\
\hline$>4.7 \mathrm{mg} . \mathrm{dL}^{-1}$ & 80.23 & 81.2 & 126 & 121 & 88 & 82 \\
\hline
\end{tabular}

Footnotes: $U A$ - uric acid; $T-C$ - total cholesterol; $T A G$ - triglycerides; $L D L$ - low-density lipoproteins; $H D L$ - high-density lipoproteins; $G$ - glucose; $B M I$ - body mass index; $V F A$ - visceral fat area; $F M$ - fat mass; $W C$ - waist circumference; $S B P$ - systolic blood pressure; $D B P$ - diastolic blood pressure 
Glucose level increased significantly during the intervention from $4.55 \pm 0.39 \mathrm{mmol} . \mathrm{L}^{-1}$ to $4.85 \pm$ 0.51 mmol.L $\mathrm{L}^{-1}(P<0.001)$. Uric acid has not changed significantly $\left(3.70 \pm 0.72 \mathrm{mg} . \mathrm{dL}^{-1}\right.$ vs $3.71 \pm 0.81$ mg.dL ${ }^{-1} ; P>0.05$ ).

Although the assessment of anthropometric parameters showed a non-significant decrease in BMI $\left(22.75 \pm 4.12\right.$ vs $\left.22.67 \pm 4.13 \mathrm{~kg} \cdot \mathrm{m}^{-2}\right)$ and fat mass (18.7 \pm 9.44 vs $18.66 \pm 9.27 \mathrm{~kg}$ ), but also an increase in visceral fat area $\left(74.10 \pm 30.16\right.$ vs $\left.74.77 \pm 31.37 \mathrm{~cm}^{2}\right)$ and waist circumference $(82.08 \pm 10.99$ vs $82.31 \pm$ $11.30 \mathrm{~cm}$ ).

In the case of blood pressure, we observed a nonsignificant decrease in systolic pressure from $121 \pm$ 9.82 to $119 \pm 9.82 \mathrm{mmHg}$ and diastolic pressure from $79 \pm 9.74$ to $77 \pm 8.61 \mathrm{mmHg}(P>0.05)$.

The monitored parameters were also evaluated by individual UA quartiles (Table 4). We found that in the case of the lipid profile, women had the highest total cholesterol, triglyceride, and LDL concentrations in the third quartile (UA between 4.1-4.6 mg.dL ${ }^{-1}$ ). The values of all the above mentioned parameters increased in the third quartile after six weeks of consumption of gluten-free bread and bakery products. In the case of HDL, the initial value was highest in the third quartile, but after the intervention in the second quartile. Linear increases in UA concentrations were not observed in any of the parameters. Similarly to other studies, in our case, the highest glucose concentrations were found in the lower UA quartiles, while the lowest in the highest quartiles.

Evaluation of the anthropometric parameters showed that while values of BMI, VFA, fat mass and waist circumference were the highest at the beginning of the study in the second quartile (UA between 3.6$4 \mathrm{mg} \cdot \mathrm{dL}^{-1}$ ), after intervention the highest values were shifted to the third quartile (UA between 4.1-4.6 $\left.\mathrm{mg} . \mathrm{dL}^{-1}\right)$. From this point of view we can conclude that due to the consumption of gluten-free bakery products the risk values of the monitored parameters shifted to higher UA quartiles.

Mean systolic blood pressure values did not exceed the limit of $140 \mathrm{mmHg}$ in any quartile of UA, with the highest values after intervention in the third quartile. The mean diastolic blood pressure values did not exceed the limit of $90 \mathrm{mmHg}$ during the study, with the highest values in the fourth quartile of UA in both cases (before and after intervention).

According to correlations we found significant relationships between total cholesterol and triglycerides, LDL, HDL, systolic and diastolic blood pressure $(P<0.01$; Table 5). Significant correlation was also found between triglycerides and LDL $(P<0.05)$, both of which had a significant relationship to systolic and diastolic blood pressure $(P<0.01)$. Correlation analysis showed a strong relationship between LDL

\begin{tabular}{|c|c|c|c|c|c|c|c|c|c|c|c|c|}
\hline & $\overrightarrow{\tilde{n}}$ & 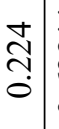 & 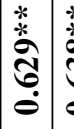 & 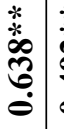 & 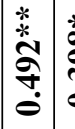 & 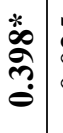 & $\mid \begin{array}{l}2 \\
\vdots \\
0 \\
0 \\
1\end{array}$ & \begin{tabular}{l|l}
$\infty$ & \\
$\circ$ \\
$\circ$ \\
$\circ$
\end{tabular} & 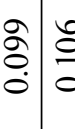 & \begin{tabular}{l|l}
$\stackrel{0}{0}$ & $\stackrel{\infty}{\circ}$ \\
\end{tabular} & 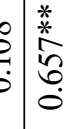 & \\
\hline & 金 & $\begin{array}{c}\hat{0} \\
\dot{0} \\
0\end{array}$ & 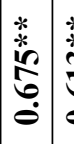 & 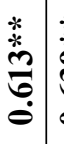 & 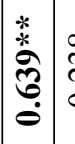 & 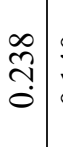 & 足 & $\begin{array}{c}\bar{\sigma} \\
\overrightarrow{0}\end{array}$ & $\begin{array}{lll}\infty & 0 \\
0 & 0 \\
0 & 0\end{array}$ & 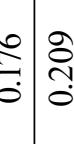 & & 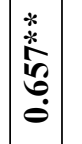 \\
\hline & 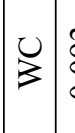 & $\begin{array}{l}\tilde{\delta} \\
\tilde{o}\end{array}$ & 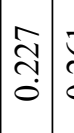 & $\begin{array}{c}\vec{\delta} \\
\stackrel{0}{\circ}\end{array}$ & 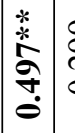 & $\begin{array}{l}\text { ते. } \\
\text { ஸे } \\
1\end{array}$ & $\begin{array}{l}\infty \\
\infty \\
\tilde{c} \\
0\end{array}$ & 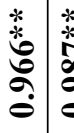 & 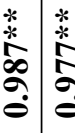 & 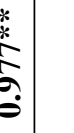 & 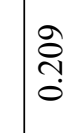 & 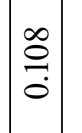 \\
\hline & $\sum_{i=}$ & $\begin{array}{l}2 \\
0 \\
0 \\
0 \\
0\end{array}$ & $\stackrel{\infty}{\infty}$ & $\frac{2}{\circ}$ & 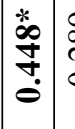 & 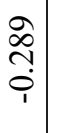 & 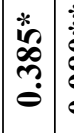 & 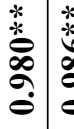 & 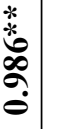 & 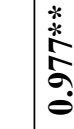 & $\stackrel{\circ}{\frac{0}{0}}$ & $\stackrel{8}{0}$ \\
\hline & $\underset{1}{>}$ & 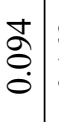 & $\frac{0}{0}$ & 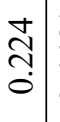 & 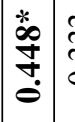 & $\begin{array}{l}m \\
m \\
\tilde{n} \\
0 \\
1\end{array}$ & 蒙 & 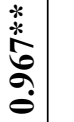 & & 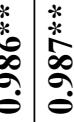 & $\frac{\infty}{0}$ & $\stackrel{\partial}{\circ}$ \\
\hline 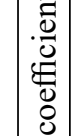 & $\sum_{m}$ & $\begin{array}{l}\stackrel{P}{0} \\
0 \\
0\end{array}$ & $\overrightarrow{\overrightarrow{0}}$ & 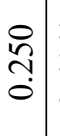 & $\stackrel{*}{\stackrel{*}{+}}$ & 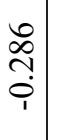 & $\begin{array}{l}\hat{q} \\
\text { ?. } \\
0\end{array}$ & & 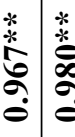 & 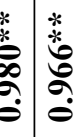 & $\begin{array}{l}\dot{0} \\
\dot{0} \\
\dot{0} \\
\dot{0}\end{array}$ & 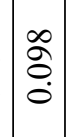 \\
\hline$\left|\begin{array}{c}0 \\
0 \\
0 \\
0 \\
0 \\
0 \\
0 \\
0\end{array}\right|$ & 0 & 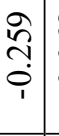 & $\mid \begin{array}{l}0 \\
0 \\
0 \\
0 \\
0 \\
1\end{array}$ & 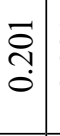 & हु & $\begin{array}{l}\stackrel{2}{\subseteq} \\
\stackrel{0}{0} \\
1\end{array}$ & & 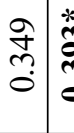 & 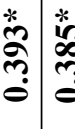 & 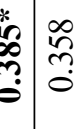 & $\begin{array}{lll}0 & 0 \\
? & 0 & + \\
0\end{array}$ & $\begin{array}{l}\tilde{a} \\
\tilde{o} \\
\dot{0}\end{array}$ \\
\hline & $\overrightarrow{⿱ 丶}$ & $\begin{array}{l}\bar{a} \\
\bar{a} \\
\dot{\varphi}\end{array}$ & 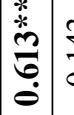 & 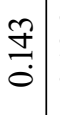 & $\mid$\begin{tabular}{l}
0 \\
$\dddot{2}$ \\
\hdashline \\
0
\end{tabular} & & $\mid \begin{array}{l}2 \\
\stackrel{2}{0} \\
0 \\
1\end{array}$ & 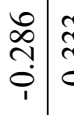 & 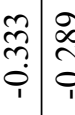 & 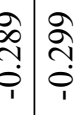 & 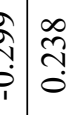 & 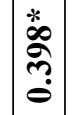 \\
\hline & $\overrightarrow{\mathrm{A}}$ & 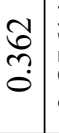 & 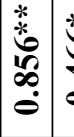 & 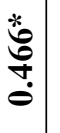 & & $\begin{array}{c}0 \\
\stackrel{2}{0} \\
\stackrel{0}{0}\end{array}$ & $\overrightarrow{\tilde{\sigma}}$ & 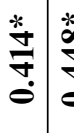 & 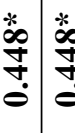 & 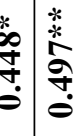 & 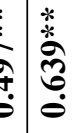 & 莣 \\
\hline & 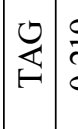 & $\begin{array}{l}\stackrel{a}{\tilde{0}} \\
\stackrel{0}{0}\end{array}$ & 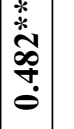 & & 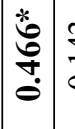 & $\frac{\mathfrak{g}}{\dot{\sigma}}$ & 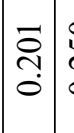 & 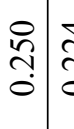 & 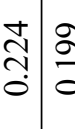 & 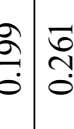 & 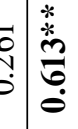 & $\left|\begin{array}{c}* \\
* \\
\infty \\
0 \\
0 \\
0 \\
0\end{array}\right|$ \\
\hline & 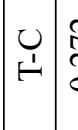 & 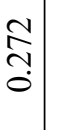 & & 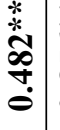 & \begin{tabular}{l}
$*$ \\
\multirow{2}{*}{} \\
$\infty$ \\
$\infty$ \\
0 \\
0
\end{tabular} & 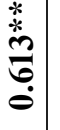 & $\mid \begin{array}{l}0 \\
0 \\
0 \\
0 \\
1 \\
1\end{array}$ & \begin{tabular}{c|c}
$\overline{0}$ \\
$\stackrel{0}{0}$ & \\
\end{tabular} & 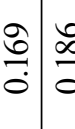 & 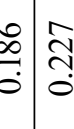 & $\begin{array}{c}v \\
v \\
s\end{array}$ & 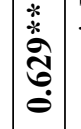 \\
\hline & 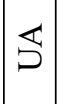 & & 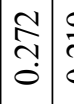 & 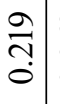 & $\begin{array}{l}0 \\
0 \\
0 \\
0\end{array}$ & $\begin{array}{l}= \\
0 \\
\vdots \\
\vdots\end{array}$ & $\mid \begin{array}{l}\hat{2} \\
\hat{1} \\
\hat{i} \\
1\end{array}$ & \begin{tabular}{l|l}
$\stackrel{R}{0}$ & \\
\hdashline & \\
\end{tabular} & 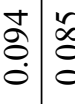 & \begin{tabular}{l|l}
0 \\
0 \\
0 \\
0
\end{tabular} & 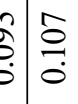 & 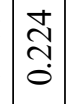 \\
\hline & & 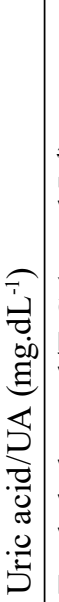 & 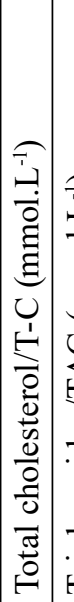 & 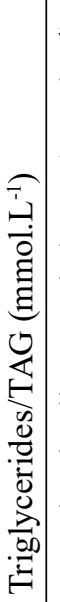 & 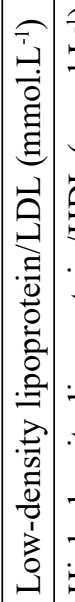 & 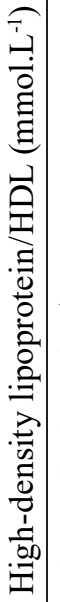 & 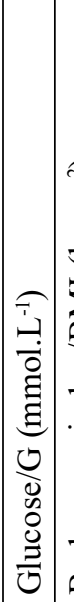 & 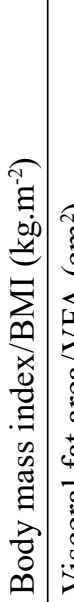 & 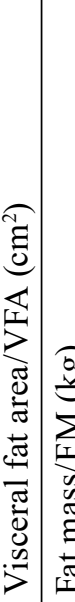 & 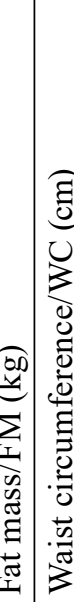 & 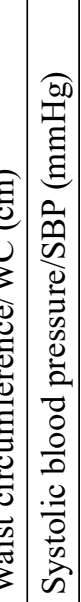 & 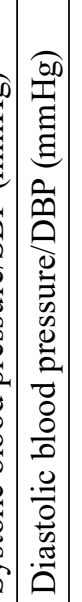 \\
\hline
\end{tabular}


and anthropometric parameters (BMI, visceral fat area, fat mass; $P<0.05$ ) and waist circumference $(P<0.01)$. Glucose had a correlation with visceral fat area and fat mass $(P<0.05)$. BMI had a significant relationship with VFA, fat mass, waist circumference $(P<0.01)$ and visceral fat area with fat mass and waist circumference $(P<0.01)$. Blood pressure systolic and diastolic was associated with lipid parameters (total cholesterol, triglycerides, LDL; $P<0.01$ ).

In terms of metabolic syndrome prevalence, at least one risk factor was found in $52 \%$ and $48 \%$ of respondents at the beginning and end of the study. Metabolic syndrome would be diagnosed in $11 \%$ of participants before intervention, but only in one case after intervention. The most female participants with one risk factor occurred in the first and fourth quartiles of the UA. Volunteers with two or more risk factors for metabolic syndrome (after intervention) were found in the second and third quartiles. Prior to intervention, the highest incidence of risk factors was in the second quartile. The most frequently occurring risk factors were increased waist circumference and diastolic blood pressure.

In terms of cardiovascular risk, total cholesterol, waist circumference, and LDL (elevated concentrations in $81 \%, 48 \%$ and $67 \%$, respectively) were the most critical parameters before and after the intervention. Most risk factors occurred in the first quartile (between 1 and 4 risk factors per participant). The highest incidence of risk factors per participant occurred in the third quartile.

The gluten-free diet is for people suffering from celiac disease, for which the only form of treatment is the strict exclusion of gluten from the diet $[9,57]$. The early development of gluten-free products has been associated with many technological and rheological problems, with the nutritional value of these products being secondary. Therefore, gluten-free products were nutritionally unbalanced [12]. Many studies have confirmed higher levels of saturated fatty acids, salt, low dietary fibre $[1,26]$. These facts support the suspicion that in such nutritional composition of glutenfree products, consumers are at risk of higher intake of risk food components involved in the development of cardiovascular, metabolic and other diseases [64].

However, considering gluten-free products suitable for weight loss could lead to overconsumption of these energy-rich products and could result in promoting weight gain [27]. Obesity is a serious health condition significantly associated with higher mortality and morbidity [30] and highly prevalent metabolic disorder that is characterized by excessive body fat mass. Abdominal obesity and excess visceral fat are independent risk factors for cardiovascular diseases [21], diabetes mellitus and total mortality [29, 51]. Visceral fat area is important factor used in the assessment of cardio-metabolic risk and is correlated with the metabolic syndrome even at the normal body mass index indicating the absence of obesity [6].

Elevated serum UA levels are commonly seen in association with individual cardiovascular and metabolic syndrome risk factors such as hypercholesterolemia, hypertriglyceridemia, hypertension, hyperglycemia, and obesity [23].

Many studies reported that the association between serum UA levels and metabolic syndrome was stronger in females than in males. Women with a higher serum UA concentration had a higher incidence of hypertension, hypertriglyceridemia and low HDL as well as increased cardiovascular morbidity and mortality compared to that in men $[7,15,24,60]$.

Yang et al. [60] observed that higher levels of serum UA were significantly associated with increasing BMI, waist circumference, systolic blood pressure, serum total cholesterol, LDL, and triglycerides but fasting plasma glucose and reduced HDL were significantly negatively related to serum UA concentration.

In the study of Zhang et al. [65] participants with metabolic syndrome were more likely to have higher levels of BMI, waist circumference, systolic and diastolic blood pressure, total cholesterol, triglycerides, fasting glucose and lower HDL levels in both men and women. The prevalence of metabolic syndrome increased according to the quartiles of serum UA concentration. In women, the prevalence raised from $24.98 \%$ among the participants with serum UA concentrations $<3.5 \mathrm{mg} . \mathrm{dL}^{-1}$ to $55.71 \%$ among those with concentrations $\geq 4.7 \mathrm{mg} . \mathrm{dL}^{-1}$. In the fourth quartile, $9.65 \%$ in women exhibited five metabolic components. There was a positive association between serum UA concentration and central obesity, hypertriglyceridemia, low HDL and high blood pressure in men and women.

The prospective study of Liu et al. [2014] conducted in US reported that subjects with a high vs low serum UA concentrations were 2.29 times more likely to have metabolic syndrome in women. In addition, the association between serum UA and metabolic syndrome was stronger among women than men, consistently with other studies [16, 54].

Hyperuricemia and hypertension may both result from the common pathway hyperinsulinemia due to insulin resistance, which increases urine sodium retention and decreases renal uric acid clearance [25].

In prospective study of a Chinese population Yang et al. [60] found that there was a graded increase in the incidence of metabolic syndrome among individuals with increasing levels of serum UA. Findings suggest that there was a sex-related association, and therefore hyperuricemia is a significantly independent risk factor for the development of metabolic syndrome in women and tended to interact additively with elevated 
blood pressure and elevated waist circumference. Postmenopausal women have higher uric acid levels than younger ones due to their lack of estrogens, which are naturally uricosuric and favour uric acid excretion. Serum UA concentrations are always lower in women than in men at any age albeit less markedly so with aging [20]. These differences are caused by other determinants as estrogens.

Zurlo et al. [67] found in their study that women with higher baseline serum UA concentrations had a higher incidence of both hypertriglyceridemia and high blood pressure than men. They also found out that high baseline serum UA concentrations were able to predict the onset of metabolic syndrome only in older women, but not in men. At the baseline, higher serum UA levels were significantly associated with more abdominal obesity in both genders and high triglyceride levels, but only in men.

Obesity is a well-recognized marker and risk factor for type 2 diabetes, but many individuals with diabetes are not obese [31]. Results of Krishnan et al. [35] suggest that elevated serum urate concentration may be one of the risk factor. Data from the Rotterdam Study showed that the age and gender adjusted hazard ratio for diabetes was greatest among persons in the highest quartile of serum urate level [22].

Hyperuricemia have been proposed as novel risk factors for diabetes, but the results from epidemiologic studies have been not clear [10, 49]. The role of serum UA as a risk factor for diabetes is gender related and due to different dietary patterns, genetic factors and the influence of sex hormones [43]. Although higher than normal serum UA levels are positively associated with diabetes, some studies suggest that patients with recently-diagnosed diabetes tend to have lower serum UA than non-diabetics [5]. Nan et al. [45] reported a bell-shape association between serum UA and fasting glucose, which showed an increasing trend in UA up to fasting glucose of $7.0 \mathrm{mmol} . \mathrm{L}^{-1}$. Thereafter, the serum UA started to decrease along with further increases in fasting glucose.

\section{CONCLUSIONS}

The results of our study showed that consumption of gluten-free bread and bakery products during six weeks had no significant effect on the observed cardiovascular and metabolic syndrome risk parameters. But we found that in the case of the lipid profile, women had the highest total cholesterol, triglyceride, and LDL cholesterol concentrations in the third quartile of UA and the values of all the above mentioned parameters increased in the third quartile after six weeks of consumption of glutenfree bread and bakery products. The highest glucose concentrations were found in the lower UA quartiles, while the lowest in the highest quartiles. In terms of metabolic syndrome prevalence, prior to intervention, the highest incidence of risk factors was in the second quartile. The most frequently occurring risk factors were increased waist circumference and diastolic blood pressure. In terms of cardiovascular risk, the highest incidence of risk factors per participant occurred in the third quartile.

\section{Acknowledgments}

This study was supported by the projects: Grant Agency of Faculty of Agrobiology and Food Resources SUA in Nitra, Slovakia (05-GA FAPZ SPU-19); KEGA no. 004SPU-4/2019 and Union of Industrial Bakers of Slovakia.

\section{Conflict of interest}

The authors declare no conflict of interests.

\section{REFERENCES}

1. Abenavoli, L., Delibasic, M., Peta, V., Turkulov, V., Lorenzo, A.D., Medić-Stojanoska, M.: Nutritional profile of adult patients with celiac disease. European Review for Medical and Pharmacological Sciences 2015;19(22):4285-4292.

2. Alberti, K.G., Eckel, R.H., Grundy, S.M., Zimmet, P.Z., Cleeman, J.I., Donato, K.A. et al.: Harmonizing the metabolic syndrome: a joint interim statement of the international diabetes federation task force on epidemiology and prevention; national heart, lung, and blood institute; american heart association; world heart federation; international atherosclerosis society; and international association for the study of obesity. Circulation 2009;120(16):1640-1645. doi: 10.1161/ CIRCULATIONAHA.109.192644

3. Alberti, K.G., Zimmet, P., Shaw, J., Group IDFETFC: The metabolic syndrome-a new worldwide definition. Lancet 2005;366:1059-1062. doi: 10.1016/S01406736(05)67402-8

4. Albrecht, E., Waldenberger, M., Krumsiek, J. et al.: Metabolite profiling reveals new insights into the regulation of serum urate in humans. Metabolomics 2014;10:141-151. doi: 10.1007/s11306-013-0565-2

5. Andrade, J.A., Kang, H.C., Greffin, S., Garcia Rosa, M.L., Lugon, J.R.: Serum uric acid and disorders of glucose metabolism: the role of glycosuria. Brazilian Journal of Medical and Biological Research 2014;47(10):917-923. doi: 10.1590/1414-431X20143878

6. Babiarczyk, B., Turbiarz, A.: Body Mass Index in elderly people - do the reference ranges matter? Progress in Health Sciences 2012;2(1):58-67.

7. Babio, N., Martínez-González, M.A., Estruch, R., Wärnberg, J., Recondo, J., Ortega-Calvo, M., SerraMajem, L., Corella, D., Fitó, M., Ros, E., Becerra-Tomás, N., Basora, J., Salas-Salvadó, J.: Associations between serum uric acid concentrations and metabolic syndrome and its components in the PREDIMED study. Nutrition, 
metabolism, and cardiovascular diseases: NMCD 2015;25(2):173-180. doi: 10.1016/j.numecd.2014.10.006

8. Balkau, B., Charles, M.A.: Comment on the provisional report from the WHO consultation: European Group for the Study of Insulin Resistance (EGIR). Diabetic Medicine: A Journal of the British Diabetic Association 1999;16:442-443. doi: 10.1046/j.1464-5491.1999.00059.x

9. Bascuñán, K.A., Vespa, M.C., Araya, M.: Celiac disease: understanding the gluten-free diet. European Journal of Nutrition 2016;56(2):449-459. doi: 10.1007/s00394-0161238-5

10.Bhole, V., Choi, J.W., Kim, S.W., de Vera, M., Choi, $H$.: Serum uric acid levels and the risk of type 2 diabetes: a prospective study. The American Journal of Medicine 2010;123(10):957-961. doi: 10.1016/j. amjmed.2010.03.027

11. Billiet, L., Doaty, S., Katz, J.D., Velasquez, M.T.: Review of hyperuricemia as new marker for metabolic syndrome. ISNR Rheumatology 2014. doi: 10.1155/2014/852954

12.Capriles, V.D., Areas, J.A.G.: Novel approaches in gluten-free breadmaking: Interface between food science, nutrition, and health. Comprehensive Reviews in Food Science and Food Safety 2014;13:871-890. doi: 10.1111/1541-4337.12091

13. Catassi, C., Bai, J.C., Bonaz, B., Bouma, G., Calabrò, $A$., Carroccio, $A$. et al.: Non-celiac gluten sensitivity: the new frontier of gluten related disorders. Nutrients 2013;5:3839-3853. doi: 10.3390/nu5103839

14. Chaudhary, K., Malhotra, K., Sowers, J., Aroor, A.: Uric acid - key ingredient in the recipe for cardiorenal metabolic syndrome. Cardiorenal Medicine 2013;3:208220. doi: 10.1159/000355405

15. Chiou, W.K., Wang, M.H., Huang, D.H., Chiu, H.T., Lee, Y.J., Lin, J.D.: The relationship between serum uric acid and metabolic syndrome: differences by sex and age in Taiwanese. Journal of Epidemiology 2010;20:219-224. doi: 10.2188/jea.je20090078

16. Choi, H., Kim, H.C., Song, B.M., Park, J.H., Lee, J.M., Yoon, D.L., Yoon, Y.M., Rhee, Y., Youm, Y., Kim, C.O.: Serum uric acid concentration and metabolic syndrome among elderly Koreans: the Korean Urban Rural Elderly (KURE) study. Archives of Gerontology and Geriatrics 2016;64:51-58. doi: 10.1016/j.archger.2016.01.005

17. Choi, H.K., Ford, E.S.: Prevalence of the metabolic syndrome in individuals with hyperuricemia. The American Journal of Medicine 2007;120(5):442-447. doi: 10.1016/j.amjmed.2006.06.040

18. Cicero, A.F., Rosticci, M., Fogacci, F., Grandi, E., D'Addato, S., Borghi, C. et al.: High serum uric acid is associated to poorly controlled blood pressure and higher arterial stiffness in hypertensive subjects. European Journal of Internal Medicine 2017;37:38-42. doi: 10.1016/j.ejim.2016.07.026

19. Conte, P., Fadda, C., Piga, A., Collar, C.: Technofunctional and nutritional performance of commercial breads available in Europe. Food Science and Technology International 2016;22:621-633. doi: $10.1177 / 1082013216637724$

20.Culleton, B.F., Larson, M.G., Kannel, W.B., Levy, D.: Serum uric acid and risk for cardiovascular disease and death: the Framingham heart study. Annals of Internal Medicine 1999;131(1):7-13. doi: 10.7326/0003-4819-1311-199907060-00003

21. De Lorenzo, A., Del Gobbo, V., Premrov, M.G., Bigioni, M., Galvano, F., Di Renzo, L.: Normal-weight obese syndrome: early inflammation? The American Journal of Clinical Nutrition 2007;85(1):40-45. doi: 10.1093/ ajen/85.1.40

22.Dehghan, A., van Hoek, M., Sijbrands, E.J., Hofman, A., Witteman, J.C.: High serum uric acid as a novel risk factor for type 2 diabetes. Diabetes Care 2008;31(2):361362. doi: $10.2337 / \mathrm{dc} 07-1276$

23.Eckel, R.H., Grundy, S.M., Zimmet, P.Z.: The metabolic syndrome. Lancet 2005;365:1415-1428. doi: 10.1016/ S0140-6736(05)66378-7

24.Fang, J., Alderman, M.H.: Serum uric acid and cardiovascular mortality the NHANES I Epidemiologic Follow-up Study, 1971-1992. JAMA 2000;283:24042410. doi: 10.1001/jama.283.18.2404

25.Forman, J.P., Choi, H., Curhan, G.C.: Uric acid and insulin sensitivity and risk of incident hypertension. Archives of Internal Medicine 2009;169(2):155-162. doi: 10.1001/archinternmed.2008.521

26.Fry, L., Madden, A.M., Fallaize, R.: An investigation into the nutritional composition and cost of gluten-free versus regular food products in the UK. Journal of Human Nutrition and Dietetics 2018;31(1):108-120. doi: 10.1111/jhn.12502

27. Gažarová, M., Chlebová, Z., Kopčeková, J., Lenártová, $P$., Holovičová, M.: The influence of gluten-free bakery products consumption on selected anthropometric parameters. Potravinarstvo Slovak Journal of Food Sciences 2017;11(1):761-769. doi: https://doi. org/10.5219/861

28.Grundy, S.M., Cleeman, J.I., Daniels, S.R., Donato, K.A., Eckel, R.H., Franklin, B.A., Gordon, D.J., Krauss, R.M., Savage, P.J., Smith Jr, S.C., Spertus, J.A., Costa, $F$. et al.: Diagnosis and management of the metabolic syndrome: an American Heart Association/National Heart, Lung, and Blood Institute Scientific Statement. Circulation 2005;112:2735-2752. doi: 10.1161/ CIRCULATIONAHA.105.169404

29.Janssen, I., Katzmarzyk, P. T., Ross, R.: Waist circumference and not body mass index explains obesity-related health risk. The American Journal of Clinical Nutrition, 79, 2004, (3), pp. 379-384. DOI: 10.1093/ajcn/79.3.379

30.Jensen, M.D., Ryan, D.H., Apovian, C.M., Ard, J.D. et al.: 2013 AHA/ACC/TOS guideline for the management of overweight and obesity in adults: a report of the American college of cardiology/American heart association task force on practice guidelines and the obesity society. Circulation 2014;129(25 Suppl):102-138. doi: 10.1161/01.cir.0000437739.71477.ee.

31. Jiang, Y., Chen, Y., Mao, Y.: The contribution of excess weight to prevalent diabetes in Canadian adults. CCDPC Obesity Working Group. Public Health 2008;122(3):271276. doi: 10.1016/j.puhe.2007.06.002

32.Kajaba, I., Štencl, J., Ginter, E., Šašinka, M.A., Trusková, I., Gazdiková, K., Hamade, J., Bzdúch, V. 
Odporúčané výživové dávky pre obyvatel'stvo SR (9. revízia). (Recommended Dietary Allowance for Slovaks.) Vestník MZ SR 2015;63(4-5):17-28, (in Slovak). file://C:/Documents\%20and\%20Settings/user/ Dokumenty/vestnik-2015-4-5.pdf

33. Kang, D.H., Chen, W.: Uric acid and chronic kidney disease: new understanding of an old problem. Seminars in Nephrology 2011;31(5):447-452. doi: 10.1016/j. semnephrol.2011.08.009

34.Kim, H.S., Patel, K.G., Orosz, E., Kothari, N., Demyen, M.F., Pyrsopoulos, N., Ahlawat, S.K.: Time trends in the prevalence of celiac disease and gluten-free diet in the us population: results from the National Health and Nutrition Examination Surveys 2009-2014. JAMA Internal Medicine 2016;176:1716-1717. doi:10.1001/ jamainternmed.2016.5254.

35. Krishnan, E., Pandya, B.J., Chung, L., Hariri, A., Dabbous, O.: Hyperuricemia in young adults and risk of insulin resistance, prediabetes, and diabetes: a 15-year follow-up study. American Journal of Epidemiology 2012;176(2):108-116. doi: 10.1093/aje/kws002

36.Kuzuya, M., Ando, F., Iguchi, A., Shimokata, H.: Effect of aging on serumuric acid levels: longitudinal changes in a large Japanese population group. The Journals of Gerontology. Series A, Biological Sciences and Medical Sciences 2002;57(10):660-664. doi: 10.1093/ gerona/57.10.m660

37. Lamacchia, C., Camarca, A., Picascia, S., Di Luccia, A., Gianfrani, C.: Cereal-based gluten-free food: How to reconcile nutritional and technological properties of wheat proteins with safety for celiac disease patients. Nutrients 2014;6(2):575-590. doi: 10.3390/nu6020575

38.Lee, J.J., Ahn, J., Hwang, J., Han, S.W., Lee, K.N., Kim, J.B., Lee, S., Na, J.O., Lim, H.E., Kim, J.W., Rha, S.W., Park, C.G., Seo, H.S., Oh, D.., Kim, E.J.: Relationship between uric acid and blood pressure in different age groups. Clinical Hypertension 2015;15(21):14. doi: 10.1186/s40885-015-0022-9

39. Leiba, A., Vinker, S., Dinour, D., Holtzman, E.J., Shani, $M$.: Uric acid levels within the normal range predict increased risk of hypertension: a cohort study. Journal of the American Society of Hypertension 2015;9(8):600609. doi: 10.1016/j.jash.2015.05.010

40.Liu, H., Zhang, X.M., Wang, Y.L., Liu, B.C.: Prevalence of hyperuricemia among Chinese adults: a national cross-sectional survey using multistage, stratified sampling. Journal of Nephrology 2014;27:653-658. doi: 10.1007/s40620-014-0082-z

41. Lucisano, M., Cappa, C., Fongaro, L., Mariotti, M.: Characterisation of gluten free pasta through conventional and innovative methods: evaluation of the cooking behaviour. Journal of Cereal Science 2012;56(3):667-675. doi: 10.1016/j.jcs.2012.08.014

42.Mazzeo, T., Cauzzi, S., Brighenti, F., Pellegrini, N.: The development of a composition database of gluten-free products. Public Health Nutrition 2014;18:1353-1357. doi: $10.1017 / \mathrm{S} 1368980014001682$

43. Meisinger, C., Döring, A., Stöckl, D., Thorand, B., Kowall, B., Rathmann, W.: Uric acid is more strongly associated with impaired glucose regulation in women than in men from the general population: the KORA F4Study. PLoS One 2012;7(5):e37180. doi: 10.1371/journal. pone. 0037180

44.Miranda, J., Lasa, A., Bustamante, M.A., Churruca, I., Simon, E.: Nutritional Differences Between a Gluten-free Diet and a Diet Containing Equivalent Products with Gluten. Plant Foods for Human Nutrition 2014;69:182-187. doi: 10.1007/s11130-014-0410-4

45.Nan, H.R., Dong, Y.H., Gao, W.G., Tuomilehto, J., Qiao, Q.: Diabetes associated with a low serum uric acid concentration in a general Chinese population. Diabetes Research and Clinical Practice 2007;76:68-74. doi: 10.1016/j.diabres.2006.07.022

46.Onat, A., Uyarel, H., Hergenc, G., Karabulut, A., Albayrak, S., Sari, I., Yazici, M., Keleş, I.: Serum uric acid is a determinant of metabolic syndrome in a populationbased study. American Journal of Hypertension 2006;19:1055-1062. doi: 10.1016/j.amjhyper.2006.02.014

47. Pasalic, D., Marinkovic, N., Feher-Turkovic, L.: Uric acid as one of the important factors in multifactorial disorders-facts and controversies. Biochemia medica 2012;22:63-75. doi: 10.11613/bm.2012.007

48.Penagini, F., Dilillo, D., Meneghin, F., Mameli, C., Fabiano, V., Zuccotti, G.V.: Gluten-free diet in children: an approach to a nutritionally adequate and balanced diet. Nutrients 2013;5(11):4553-4565. doi: 10.3390/ nu5114553

49.Pfister, R., Barnes, D., Luben, R., Forouhi, N.G., Bochud, M., Khaw, K.-T., Wareham, N.J., Langenberg, $C .:$ No evidence for a causal link between uric acid and type 2 diabetes: a Mendelian randomisation approach. Diabetologia 2011;54(10):2561-2569. doi: 10.1007/ s00125-011-2235-0

50.Ravaglia, G., Forti, P., Maioli, F., Bastagli, L., Chiappelli, M., Montesi, F., Bolondi, L., Patterson, $C$ : Metabolic syndrome. Prevalence and prediction of mortality in elderly individuals. Diabetes Care 2006;29:2471-2476. doi: 10.2337/dc06-0282

51. Seidell, J.C., Perusse, L., Despres, J.P., Bouchard, C.: Waist and hip circumferences have independent and opposite effects on cardiovascular disease risk factors: the Quebec Family Study. The American Journal of Clinical Nutrition 2001;74(3):315-321. doi: 10.1093/ ajcn/74.3.315

52.Shani, M., Vinker, S., Dinour, D., Leiba, M., Twig, G., Holtzman, E.J., Leiba, A.: High normal uric acid levels are associated with an increased risk of diabetes in lean, normoglycemic healthy women. The Journal of Clinical Endocrinology and Metabolism 2016;101(10):37723778. doi: 10.1210/jc.2016-2107

53. So, A., Thorens, B.: Uric acid transport and disease. The Journal of Clinical Investigation 2010;120(6):1791-1799. doi: $10.1172 / J C I 42344$

54.Sui, X., Church, T.S., Meriwether, R.A., Lobelo, F., Blair, S.N.: Uric acid and the development of metabolic syndrome in women and men. Metabolism 2008;57:845852. doi: 10.1016/j.metabol.2008.01.030

55. Vaziri, N.D., Freel, R.K., Hatch, M.: Effect of chronic experimental renal insufficiency on urate metabolism. 
Journal of the American Society of Nephrology 1995;6:1313-1317.

56.Viazzi, F., Rebora, P., Giussani, M., Orlando, A., Stella, A., Antolini, L., Valsecchi, M.G., Pontremoli, $R$., Genovesi, S.: Increased serum uric acid levels blunt the antihypertensive efficacy of lifestyle modifications in children at cardiovascular risk. Hypertension 2016;68(5):934-940. doi: 10.1161/ HYPERTENSIONAHA.115.06852

57. Vici, G., Belli, L., Biondi, M., Polzonetti, V.: Gluten free diet and nutrient deficiencies: A review. Clinical Nutrition 2016;35(6):1236-1241. doi: 10.1016/j. clnu.2016.05.002

58. Wei, F., Sun, N., Cai, C., Feng, S., Tian, J., Shi, W., Xu, W., Wang, Y., Yang, X., Li, W.D.: Associations between serum uric acid and the incidence of hypertension: a Chinese senior dynamic cohort study. Journal of Translational Medicine 2016;14(1):010. doi: 10.1186/ s12967-016-0866-0

59. Wu, C.Y., Hu, H.Y., Chou, Y.J., Huang, N., Chou, Y.C., Lee, M.S., Li, C.P.: High serum uric acid levels are associated with all-cause and cardiovascular, but not cancer, mortality in elderly adults. Journal of the American Geriatrics Society 2015;63(9):1829-1836. doi: 10.1111/jgs.13607

60.Yang, T., Chu, C.H., Bai, C.H., You, S.L., Chou, Y.C., Chou, W.Y., Chien, K.L., Hwang, L.C., Su, T.C., Tseng, C.H., Sun, C. A.: Uric acid level as a risk marker for metabolic syndrome: a Chinese cohort study. Atherosclerosis 2012;220(2):525-531. doi: 10.1016/j. atherosclerosis.2011.11.014.

61. Yokoi, Y., Kondo, T., Okumura, N., Shimokata, K., Osugi, S., Maeda, K., Murohara, T.: Serum uric acid as a predictor of future hypertension: stratified analysis based on body mass index and age. Preventive Medicine 2016;9(90):201-206. doi: 10.1016/j.ypmed.2016.07.007
62.Yoo, T.W., Sung, K.C., Shin, H.S., Kim, B.J. et al.: Relationship between serum uric acid concentration and insulin resistance and metabolic syndrome. Circulation Journal 2005;69:928-933. doi: 10.1253/circj.69.928

63. Yu, T.Y., Jee, J.H., Bae, J.C., Jin, S.M., Baek, J.H., Lee, M.K., Kim, J.H.: Serum uric acid: A strong and independent predictor of metabolic syndrome after adjusting for body composition. Metabolism 2016;65(4):432-440. doi:10.1016/j.metabol.2015.11.003

64.Zanini, B., Mazzoncini, E., Lanzarotto, F., Ricci, C., Cesana, B.M., Villanacci, V., Lanzini, A.: Impact of gluten-free diet on cardiovascular risk factors. A retrospective analysis in a large cohort of coeliac patients. Digestive and Liver Disease 2013;45(10):810815. doi:10.1016/j.dld.2013.04.001

65.Zhang, H., Li, Y., Mao, Z., Liu, X., Zhang, X., Yang, K., Liu, R., Qian, X., Zhang, H., Jiang, J., Zhang, G., Wang, $C .:$ Sex-specific associations of serum uric acid with metabolic syndrome in Chinese rural population: The RuralDiab study. Clinica Chimica Acta 2018;480:119125. doi: 10.1016/j.cca.2018.02.003

66.Zuccotti, G., Fabiano, V., Dilillo, D., Picca, M., Cravidi, $C$., Brambilla, $P$.: Intakes of nutrients in Italian children with celiac disease and the role of commercially available gluten-free products. Journal of Human Nutrition and Dietetics 2013;26(5):436-444. doi: 10.1111/jhn.12026

67.Zurlo, A., Veronese, N., Giantin, V., Maselli, M., Zambon, S., Maggi, S., Musacchio, E., Toffanello, E.D., Sartori, L., Perissinotto, E., Crepaldi, G., Manzato, E., Sergi, G.: High serum uric acid levels increase the risk of metabolic syndrome in elderly women: The PRO.V.A study. Nutrition, Metabolism \& Cardiovascular Diseases 2016;26(1):27-35. doi: 10.1016/j.numecd.2015.10.007

Received: 05.06.2020

Accepted: 07.07.2020 